\title{
Extending the Reach and Task-Shifting Ophthalmology Diagnostics Through Remote Visualisation
}

Mario E. Giardini, lain A. T. Livingstone

This is a post-peer-review pre-copyedit author-generated version of: Giardini M.E., Livingstone I.A.T. (2020) Extending the Reach and Task-Shifting Ophthalmology Diagnostics Through Remote Visualisation. In: Rea P.M. (eds) Biomedical Visualisation. Advances in Experimental Medicine and Biology, vol 1260. Springer, Cham.

The final authenticated version is available at https://doi.org/10.1007/978-3-030-47483-6_9. 


\section{Extending the Reach and Task Shifting Ophthalmology Diagnostics Through Remote Visualisation}

Mario E. Giardini and lain A. T. Livingstone

\begin{abstract}
Driven by the global increase in the size and median age of the world population, sight loss is becoming a major public health challenge. Furthermore, the increased survival of premature neonates in low- and middle-income countries is causing an increase in developmental paediatric ophthalmic disease. Finally, there is an ongoing change in health-seeking behaviour worldwide, with consequent demand for increased access to healthcare, including ophthalmology. There is therefore the need to maximise the reach of resourcelimited ophthalmology expertise in the context of increasing demand. Yet, ophthalmic diagnostics critically relies on visualisation, through optical imaging, of the front and of the back of the eye, and teleophthalmology, the remote visualisation of diagnostic images, shows promise to offer a viable solution.

In this chapter, we first explore the strategies at the core of teleophthalmology and, in particular, real-time vs store-and-forward remote visualisation techniques, including considerations on suitability for different tasks and
\end{abstract}

M. E. Giardini

Department of Biomedical Engineering, University of

Strathclyde, Glasgow, Scotland, UK

e-mail: mario.giardini@strath.ac.uk

I. A. T. Livingstone $\left(^{*}\right)$

NHS Forth Valley, Larbert, Scotland, UK

e-mail: iain.livingstone@nhs.net environments. We then introduce the key technologies suitable for teleophthalmology: anterior segment imaging, posterior segment imaging (retinal imaging) and, briefly, radiographic/tomographic techniques. We highlight enabling factors, such as high-resolution handheld imaging, high data rate mobile transmission, cloud storage and computing, 3D printing and other rapid fabrication technologies and patient and healthcare system acceptance of remote consultations. We then briefly discuss four canonical implementation settings, namely, national service provision integration, field and community screening, optometric decision support and virtual clinics, giving representative examples. We conclude with considerations on the outlook of the field, in particular, on artificial intelligence and on robotic actuation of the patient end point as a complement to televisualisation.

\section{Keywords}

Teleophthalmology - Remote visualisation • Teleconsultation $\bullet$ Store-and-forward $\bullet$ Retinal imaging - Anterior segment imaging • Virtual clinics 


\subsection{Background}

The world population is in constant growth. From 7.7 billion in 2019, it is expected to reach 9.8 billion in 2050 , with a current growth rate of 83 million/year (United Nations 2019). Simultaneously, the life expectancy is rapidly increasing. In 2015, the population aged 60 and over, or 80 or older, was 600 million and 125 million respectively, and is expected to increase to 2 billion and 430 million, respectively, by 2050 , with $80 \%$ of these living in low- and middle-income countries (World Health Organization 2018). One of the biggest challenges of healthcare provision in the twenty-first century is therefore to find innovative solutions to build capacity, yet without sacrificing the quality of care, in a context of a globally decreasing resource-to-demand ratio.

In lower income countries, eye diseases such as river blindness and trachoma, constitute the primary cause of sight loss. In higher-income countries, other population-level threats to eyesight are emerging. Driven by lifestyle changes and age, diabetes is increasing amongst population groups, both old and younger, and diabetic retinopathy has been added to the priority list for visual impairment of the World Health Organization. Glaucoma remains a public health priority due to its complex early diagnosis and its requirements for lifelong treatment. The prevalence of age-related macular degeneration (AMD), currently $8.7 \%$ globally, is rapidly increasing, and is now the first age-related cause of visual impairment in high-income countries (World Health Organization 2019a).

In 2010, the International Council of Ophthalmology conducted a survey to establish, on a global scale, the size of the population needing ophthalmic services, the number of ophthalmologists in practice and training and the related temporal trends (Resnikoff et al. 2012). The survey highlighted that, globally, the ophthalmic population is growing faster than the number of ophthalmologists, albeit somewhat slower than the general population. Amongst those aged 60 or more, the growth rate is double than that of the number of available ophthalmologists. In the current service delivery model, a shortfall in the number of practising ophthalmologists is therefore expected, both in high- and in low-income countries. Indeed, already at present, there is a clear inverse correlation between the prevalence of visual impairment and number of locally available ophthalmologists (Bastawrous and Hennig 2012) indicating that the incidence of visual impairment is resource-limited by eye care availability.

Additionally, health-seeking behaviour (HSB) is rapidly increasing, with direct impact on service utilisation. Population and healthcare systems characteristics and external environment, including social and economic determinants, are key influencing factors (Mackian et al. 2004). While the modelling of the interactions between these factors and service utilisation is complex, it is clear that, on a global scale, there is a trend towards an HSBdriven increase on the global service utilisation burden, both in high- and in low-income countries and across multiple medical disciplines (Clewley et al. 2018; Shahik and Hatcher 2004;Ahmedetal. 2000). Ophthalmology is no exception, and the understanding of the interrelation between the perceived severity of manifest conditions and the necessity to proactively seek eye health is common both to high- and low-income countries (Ebeigbe 2018; Fallatah 2018).

Finally, worldwide $10 \%$ of births are preterm. Yet, oxygen administration in incubators, while necessary for survival, can induce anomalous retinal vessel growth (retinopathy of prematurity, ROP), ultimately resulting in complete retinal detachment and consequent blindness. Incidence is difficult to estimate in a single comprehensive figure, as it critically depends on gestational age at birth, weight at birth, preterm management protocols and survival rates amongst other factors. For reference, incidences of $25-35 \%$ of severe ROP in infants with a gestational age at birth of 27 weeks or less have been reported in high-income countries (Hellstrom et al. 2013) and, in 2010, approximately 170,000 preterm infants developed any stage of ROP globally, of which 20,000 became blind or severely visually impaired, and a further 12,000 mildly/moderately impaired (Blencowe et al. 2013). Importantly, $65 \%$ of ROP-related visually impaired infants were born in middle-income countries, where survival rates are increasing due to improving perinatal care (Freitas et al. 2018). Similar growth trends are reported in high-income countries (Holmstrom et al. 2018).

It is therefore clear that there is a need to maximise the reach of resource-limited ophthalmology expertise in a context of increasing demand. Beyond 
the resource limitations inherently encountered in low-income countries, also in high-income countries, a clear, direct link between scarcity of ophthalmology resources and social deprivation is beginning to emerge. As early as 2014, electronic connectivity was brought forward in policy recommendations by several UK charities as a viable solution to extend the reach of ophthalmology services, for the provision of accessible, equitable and timely integrated access to eye services (The College of Optometrists 2014).

\section{$9.2 \quad$ Strategies}

In a client-to-provider context, the World Health Organization recommends telemedicine as a complement, rather than a replacement, to face- toface healthcare delivery, with institutional monitoring of standard operating procedures, patient consent collection, data protection and provider licensing and credentials. Provider-to- provider telemedicine is seen as a method to extend coverage for individual healthcare providers (World Health Organization 2019b). In both cases, telemedicine consultations can proceed in two modalities. In realtime telemedicine, the remote ('doctor') and local ('patient') sites can be connected through a direct video and audio feed, enabling live interaction between doctor and patient. Alternatively, in storeand-forward (asynchronous) telemedicine, information, such as health records, radiology scans and other imaging diagnostics, and test results, is gathered at the local site, and transmitted to, or retrieved by, the remote site at a later time, for review when the patient is no longer present.

Both modalities are employed in the widest range of environments from clinics to community care to field medicine. The use cases are, however, significantly different, as the two approaches imply significantly different prerequisites in the need for patient presence. Real-time telemedicine is suitable to provide remote consultation to patients who require immediate advice. To all intents, it aims to replace in-person meetings, reducing the need for the patient or physician to travel to a separate facility, for example, for a separate consultation (AMD Global Telemedicine 2019). Akin to a traditional face-to- face consultation, it allows the clinician to explore additional history or examination findings and enables dialogue between the clinician, referrer and/or patient.

Conversely, as entirely reliant on qualified data collection and interpretation, store-and- forward consultation are predominantly used for doctordoctor interactions, with significant prevalence in radiology, pathology, dermatology and ophthalmology (National Center for Connected Health Policy 2019a).

Unsurprisingly, therefore, the two techniques, if used within the same context, can yield significantly different outcomes. Comparative studies in teledermatology highlight that, when used without use case differentiation, real-time and store-andforward approaches agree in half of cases only, with an agreement in the resultant treatment plans in less than half. This significant discrepancy has been directly attributed to the absence of patient-doctor interaction in store- and-forward approaches, which, while significantly cheaper than real time, constrains the ability to gather clinically relevant useful information (Loane et al. 2008). Yet, the same study highlights how, on the same cases, store-andforward paradigms require an order of magnitude less time than real-time consultations, and this reduction in time may arguably act as confounding factor in reliability analysis. With specific regard to teleophthalmology, the evidence base for an effectiveness comparison between store- andforward and real-time consultations is fragmented. Yet, the landscape of teleophthalmology trials is active and dynamic globally, on a very reasonable presumptive basis, given the prerequisites (Sim et al. 2016; Sreelatha and Ramesh 2016).

Understandably, reimbursement rates and policies regulating the two approaches are significantly different, with both private insurers and public legislation favouring real-time patientdoctor video consultations over store-and- forward doctor-doctor, or non-video real-time interaction (e.g. phonebased), that are considered inherently less expensive, and in most instances, already intrinsically integrated in the current workflows as a matter of fact (National Center for Connected Health Policy 2019b). 


\section{$9.3 \quad$ Technologies andFactors}

\subsubsection{Eye Visualisation Technologies}

Traditionally, ophthalmology critically relies on the visualisation of eye structures, supported by a set of auxiliary measurements (intraocular pressure, corneal topography, etc.) and by functional tests (e.g. visual acuity and visual field tests). Within teleophthalmology, imaging constitutes the first-line approach and can be broadly divided into three core families. In anterior segment imaging, optical images of the components of the eye directly visible from a general external inspection (eyelids, conjunctiva, cornea, sclera, anterior chamber, iris and lens) are imaged optically through some form of microscope or closeup inspection lens. In posterior segment imaging, visual access to the retina is gained through the pupil, and the retina is visualised through the lens using appropriate optics, such as an ophthalmoscope or a retinal camera. In radiographic and tomographic techniques (Optical Coherence Tomography - OCT, ultrasound imaging and largescale tomography) images are derived through computer reconstruction rather than by direct optical visualisation.

Potentially, all these imaging modalities lend themselves to remote transmission and visualisation of images, under the prerequisite that the related instrumentation needs to be compatible in cost, ruggedness and ease of use for deployment at the specific patient locations and for operation by a nonspecialist when required.

Both anterior and posterior segment imagers can be implemented using full-featured fullperformance instruments or task-specific lowcost, low-skill instrumentation, as required by the specific use cases.

Anterior segment imaging is traditionally performed in the clinic using a binocular slit lamp (Carl Zeiss Meditec AG n.d.). The slit lamp design has remained essentially unchanged since its invention in 1911 (Timoney and Breathnach 2013). The patient rests their head on a head-and- chin rest, and the ophthalmologist looks at the anterior segment using a long-working-distance stereomicroscope. The illumination is provided by a light source that projects light onto the eye, and that can be sized/rotated to illuminate the eye from different directions. Additionally, for the visualisation of de- epithelialised lesions, fluorescent eye drops can be applied to the eyes, the eye illuminated with an auxiliary blue light.

Given that the slit lamp is essentially a stereomicroscope with a highly specialised light source, digital images can easily be captured either through a digital microscope camera port or by attaching the camera to the slit lamp eyepieces via add-on adapters. While these solutions indeed can provide an easy path to remote visualisation, for example, by transmitting the video feed or the images captured by the camera through commercial videoconferencing software, at the patient side the eyes are in any case visualised using a slit lamp, thus requiring an appropriately trained operator. Yet, in emergency and field ophthalmology, the majority of necessary anterior segment imaging information can be obtained, much more simply, by observation through a magnifying glass. Indeed, emergency teleophthalmology in the field has been demonstrated using nothing more than a simple macro lens attached to a mobile phone (Ribeiro et al. 2014). Dedicated anterior segment imagers working on this principle, comprising a macro lens, a white/blue light source and a battery, are now commercially available as mobile phone add-ons (Eidolon Optical 2019; Tuteja 2016).

The optical visualisation of the retina (posterior segment imaging) is somewhat more complex. Indeed, the sole optical access to the retina is through the pupil, effectively a small hole at the centre of the iris. Yet, by its intrinsic nature, the retina lies on the focal surface of the imaging optical elements of the eye (primarily cornea and lens). This means that the retina is optically conjugate to infinity or, in other words, if we were able to look directly into a subject's pupil, we would see the retina as a large surface at infinite distance. Indeed, the simplest way to look at the retina is for the ophthalmologist to move very close to the eye, limited only by the contact with the patient, and to inspect the retina through the pupil using an appropriate device to illuminate the interior of the eye. This technique, known as direct ophthalmoscopy, has been the mainstay for posterior segment visualisation since its invention by Hermann von Helmoltz in 1851, upon prior studies by Charles Babbage (Keeler 2003). As the retina is being visualised through the tiny aperture of the pupil, the field of view is small, akin to visualising the interior of a darkened room through 
the keyhole of the door. Even when dilating the pupil pharmacologically, the angular aperture does not exceed $5-10^{\circ}$.

When a larger field is desired, a large converging lens with short focal length can be interposed in front of the eye, creating a wide-field image of the retina through the pupil, which in turn can be observed using optical instruments (indirect ophthalmoscopy) (Bass 2009). Using this strategy, a slit lamp can indeed be employed also for posterior segment imaging. In order to do this, the converging lens is held by hand between the slit lamp and the eye, creating an image of the retina through the pupil, which is then inspected using the slit lamp microscope. Alternatively, instead of the slit lamp, an imaging system, effectively a dedicated magnifying loupe with illuminator, can be worn head-mounted by the ophthalmologist, again holding by hand the converging lens in front the patient. In both cases, the alignment and mutual distances between patient, lens and slit lamp/head-mounted optics are critical, and the technique requires significant skill and training to master, tending to be limited to secondary/tertiary care. Simplified monocular versions can be miniaturised as handheld devices, reducing the skills required by somewhat sacrificing the field of view, which reduces to approximately $25^{\circ}$ (Welch-Allyn 2019a). To further simplify and deskill the procedure, in principle, the microscope and lens can be rigidly mounted on a frame. Indeed, this is the operating principle of retinal cameras, whereby a self-contained unit encompasses the converging lens, the light source, the microscope and a (digital) camera for image acquisition. The field of view of retinal cameras can be on the order of $90^{\circ}$. These devices, albeit expensive and requiring a tabletop stationary mount, are sufficiently user friendly to be operated by lay personnel with simple spot training.

Due to the significant skills required at the patient end, slit lamps and head-mounted binocular indirect ophthalmoscopes are generally not suitable for community-based teleophthalmology, where they are operated by lay operators. Retinal cameras, in providing a direct digital image feed, can be easily interfaced to data transmission and teleconferencing software, and have indeed been employed in teleophthalmology trials, including trials in austere environments and using lay operators (Bastawrous 2014). For low- and middle-income settings, major downsides relate to the cost of the instruments, their size and weight and the need for a connection to mains electricity, often problematic in the field. Yet, handheld teleophthalmology-enabled devices can be obtained by using either direct or monocular indirect ophthalmoscopes, and replacing the operator's eye with the camera of a mobile phone. The accommodating power of the operator's eye, necessary for these devices to function is, in this case, replaced by the autofocus features of the phone camera. Indeed, monocular handheld indirect ophthalmoscopes, when used with a mobile phone, can yield similar fields of view than when used by direct vision (Welch-Allyn 2019b). Interestingly, the phone camera can be used to replace not only the operator's eye, but also to act as the full imaging microscope/loupe optics. In this case, the sole optical element required to perform indirect ophthalmoscopy using a phone is the large short focal converging lens that is held in front of the patient's eye. Indeed, commercial implementations of this principle exist, with intermediate field between 'standard' handheld monocular indirect ophthalmoscopes and retinal cameras. (oDocs Eye Care 2019a). Interestingly, in direct ophthalmoscopy, the simplest posterior segment imaging technique, the field of view is essentially limited by the ophthalmologist and the patient coming into contact or, in other words, by the minimum distance between the operator and the patient's eyes. Yet, a phone camera can be brought significantly closer to the patient's eye than the eye of a human operator. For this reason, several phonebased direct ophthalmoscopes have been reported on the market, with field of view comparable to monocular handheld indirect ophthalmoscopes (Giardini et al. 2014; Tuteja 2016; D-Eye 2019).

Tomographic techniques have also been reported in teleophthalmology, using both OCT and ultrasonography (Kelly et al. 2011; Lapere 2018). As these imaging techniques are computerbased, they intrinsically lend themselves to remote visualisation both through computer screen sharing and through storage and retransmission of screenshots and images. In combination with multimodal ultra-widefield imaging, OCT imaging enables assessment of a range of retinal diseases (macular degeneration, diabetic retinal disease). Such medical retina Virtual Clinics present a popular option within western Hospital Eye Services (Lee et al. 2018), where 
networked systems afford an opportunity for asynchronous diagnosis and management.

\subsubsection{Enabling Factors}

While, from a conceptual point of view, teleophthalmology relies essentially on simple remote visualisation of digital images, the practical deployment of clinically meaningful implementations relies on the convergence between a key set of core technologies, policies and cultural aspects, which only recently are appearing as sufficiently mature for realistic impact on healthcare service.

\subsubsection{High-Resolution Handheld Imaging}

Remote visualisation solutions for teleophthalmology are ideally compact to minimise the need for dedicated facilities during field deployment. In this sense, low-cost miniaturisation of the camera, the sensing element at the core of all non-tomographic ophthalmic imaging and of the digital displays for local visualisation (viewfinders, monitors) has proven to be a key enabling factor. The early development of teleophthalmology for community screening has indeed been driven by the mobile phone market (Bolster et al. 2014). While no formal theory is available on the actual resolution requirements, it is the experience of the authors that a 3-megapixel resolution is appropriate for meaningful still images during field screening. Indeed, in the early 2010s, the availability of cameras with resolution in the megapixel range on board of mainstream smartphones has coincided with the commercial or pre-commercial availability of smartphone adapters for direct and indirect ophthalmoscopy (Peek Vision 2019; Welch Allyn 2019b; D-Eye 2019; oDocs Eye Care 2019a). In terms of actual instrumental complexity, we note that the autofocus system of modern smartphone cameras can adjust the imaging optics well beyond the capabilities of the range of accommodation of a human eye, thus enabling the capture of images and video through traditional optical arrangements for slit lamps and ophthalmoscopes with little to no modification, bar simple mechanical adaptation of the phone to the instrument eyepieces (Loomba et al.

\subsubsection{High Data Rate Mobile Interconnections}

While still images can indeed relay meaningful clinical information from the field to a remote ophthalmologist, most screening and diagnostic visualisation protocols rely on some form of realtime observation of the patient's eyes. In a comprehensive teleophthalmology solution, video streaming capabilities are therefore highly desirable. Also in this case, no formal theory is available on the actual resolution, frame rate and image compression requirements. It is, however, direct experience of the authors that such parameters are less important than may have been expected, as long as the video communication is comparable to the quality normally attainable using mainstream Internet video calling software. Arguably, this video quality enables all examinations of the front and back of the eye, as long as no direct visualisation of individual cells floating in the transparent components of the eye is required, as is observed in forms of uveitis. As resolution of camera sensors and data transmission technology evolves, the limitations on cell visualisation are expected to improve.

It has been our experience that $3 \mathrm{G}$ data connectivity is sufficient for this level of performance. Again, mobile phones appear as the ideal technology platform. Starting from the last decade, mobile phones integrate a camera sensor with appropriate resolution, sophisticated autofocus optics, a highresolution display and a full data telecommunications system. Given the extreme global ubiquity of mobile phone technology, the globally high level of mobile phone literacy, and the globally available mobile infrastructure (GSM Association 2019), mobile telephony has been identified as the key teleophthalmology enabler for community-based screening programmes on a global scale (Bolster et al. 2014; Giardini 2015).

\subsubsection{Cloud Technologies}

Teleophthalmology is clinically meaningful when in-person consultations between doctor and patient would not be reasonably viable. In this sense, for community-based teleophthalmology, there is often a requirement for the patient-side end point of the connection to operate in remote geographical areas, with data connectivity of poor quality. In typical 
community screening scenarios, flexibility needs therefore to be provided to adapt the data exchange between the teleconsultation end points to the respective local network conditions as a matter of course, for example, through buffering and temporary storage, effectively atypical forms of store-and- forward. To our knowledge, comprehensive commercial solutions are still unavailable, and the largest field teleophthalmology campaigns have resorted to custom implementations (Bastawrous et al. 2016).

The necessity to process the data streams through appropriate cloud infrastructure, rather than through a mere end-to-end teleconference, offers the opportunity to implement forms of automated analysis of the image streams. Indeed, in the specific case of diabetic retinopathy screening, the most robustly evaluated canonical example of teleophthalmology (Surendran and Raman 2014), there is supporting evidence that automatic prescreening for obviously non-diseased retinal images is safe, thus reducing the workload for expert human graders (Fleming et al. 2011), with machine learning classification techniques, a central part of Scotland's diabetic retinal screening network for over 10 years.

\subsubsection{Rapid Fabrication Technologies}

Given the close interconnection between the evolution of mobile telephony devices and the development of handheld teleophthalmology solutions, the very rapid life cycle of commercial smartphone models puts the corresponding teleophthalmology devices under very high pressure to adapt to smartphone market evolutions and to avoid obsolescence. Indeed, at the time of writing, several of the mainstream phone-based retinal imaging systems are reliant on smartphones no longer available commercially or at the end of their commercial life cycle (D-Eye 2019; Welch- Allyn 2019b; Volk 2019). For this reason, a number of manufacturers of handheld ophthalmology devices have resorted to rapid fabrication in the initial preproduction studies (Bastawrous et al. 2016), at times maintaining this approach well into commercial distribution (oDocs Eye Care 2019b).

In at least one notable case, the commercial implementations of the teleophthalmology devices have been pursued in parallel with an Open Source distribution model, whereby endusers can either buy the instruments or download the full component design from an online repository, and leveraging consumer-grade low-cost rapid fabrication technology or purchasing a prefabricated kit, can self-assemble a smartphonebased retinal imager (oDocs Eye Care 2019b).

While the humanitarian reasons for this approach can easily be understood, to our knowledge there is currently no established pathway for opensource self-fabricated devices to comply with relevant regulatory constraints for human medical use, unless appropriate quality systems are put in place around the full design and fabrication processes. Yet, the democratisation of design enabled by low-cost rapid prototyping, combined with the Open Source ethical drive towards co-creation of technologies, sharing of ideas, designs, test criteria, safety and performance data, appears optimally suited to tackle major global healthcare challenges. Indeed, strategic effort is being dedicated by key international organisations to create a viable infrastructure and methodology for the democratic development of open-source healthcare solutions (UBORA 2019).

\subsubsection{Patient and Healthcare System Acceptance of Remote Consultations}

The evidence base on the acceptance of telemedicine by the clinician and patient communities provides conflicting information, with examples such as the USA, where the number of telemedicine consultations exceeds in-person consultations for key healthcare providers (Owens 2018), and South Korea, where, as of 2014, telemedicine was deemed illegal (Rho et al. 2014). Perceived ease of use and perceived usefulness have been identified as the key determinant factors in stakeholder acceptance of telemedicine (Yu et al. 2009; Rho et al. 2014). In this sense, teleophthalmology is no exception. While no systematic study is available to our knowledge to date, cohort observations report very high satisfaction rates both amongst doctors and clinicians (Grisolia et al. 2017; Rani et al. 2006; Poyser et al. 2019). Indeed, in selected countries, the national healthcare infrastructure is investing in teleconsultation services, with the implementation of dedicated software platforms that, in addition to conventional videoconferencing capabilities, allow for the virtualisation of the full consultation journey, for example, by providing virtual waiting rooms and queuing mechanisms, indicating that the core 
stakeholders are now shifting towards telemedicine as an accepted working practice (Attend Anywhere 2019; NHS Near Me 2019). This coincides with our experience implementing teleophthalmology in emergency services in Scotland.

\section{$9.4 \quad$ Implementation Examples}

\subsubsection{National Healthcare Services: Emergency Teleophthalmology in NHS Scotland}

Healthcare in Scotland is provided by the National Health Service (NHS), publicly funded through taxation. The primary care backbone of eye care in Scotland is constituted of optometrists operating in community practices (Jonuscheit 2019). Secondary care in Scottish ophthalmology departments exceeds 400,000 cases per year, with an annual increase close to $15 \%$. The entry point for emergency eye care is provided by general Accident and Emergency (A\&E) and Minor Injuries Units (MIU), where unscheduled patients are first seen by a nonspecialist healthcare operator, who then decides, often assisted by a phone consultation with an oncall ophthalmologist, whether to refer the patient to an available ophthalmology department, where the patient will need to transfer, wait for the specialist to be available and receive specialist care. Given the nature of the Scottish territory, where significant segments of the population live in remote areas, this second immediate emergency referral, often requested by the non-specialist A\&E staff on a precautionary basis only, is onerous both for the patient and the NHS, with inappropriate referrals creating an avoidable burden upon the Service.

At the time of writing, there is paucity in the literature regarding real-time applications of teleophthalmology, despite evolving examples of realtime video transmission as a novel solution in lowresource settings, for the screening of rural populations (Loomba et al. 2019). In 2017, under direct funding by the Scottish Government, the NHS Forth Valley Health board, based in Central Scotland, in collaboration with the University of Strathclyde, Glasgow, developed hardware elements and implemented a pilot teleophthalmology pipeline aimed towards optimising triage via remote decision support, reducing the waiting times and providing real-time feedback from ophthalmology specialists to emergency services, with a view to reducing the number of unnecessary specialist referrals from the A\&E departments as well as unnecessary urgent A\&E visits by ophthalmic staff. Using the Web browser-based tele-consultation platform of NHS (NHS Near Me 2019), the scheme uses two-way real-time audio-visual communication between the on-call ophthalmologist and the patient and staff in the A\&E unit through a tablet computer. Specially designed ergonomic adapters connect the tablet computer to the ophthalmology equipment (slit lamps) in the A\&E or MIU, for the ophthalmologist to visualise the patient's eyes, and to give instructions to the A\&E staff, thus allowing in-depth triage and institution of simple therapy.

To the date of writing, the 2017 pilot has been successfully completed, with an estimated 50\% reduction in emergency referrals to specialist emergency ophthalmology services. The technology has been introduced into practice and is now standard protocol in the Forth Valley NHS board. In 2019, the Scottish Government has agreed to fund the extension of the initial pilot to the NHS Highlands, NHS Grampian and NHS Greater Glasgow and Clyde health boards, to gather the evidence base for an eventual national roll-out and to evaluate the applicability of further phonebased handheld retinal imaging solutions. Modifications of the protocol are also undergoing seminal concept studies, to expand the reach of the service to community optometrists, involving both slit lamp real-time and OCT offline imaging. In view of potential extensions of the methodology to higher-resolution imaging, a $4 \mathrm{~K}$ video consultation, utilising a $5 \mathrm{G}$ mobile data network, has been demonstrated using a modified version of the NHS Scotland tele-consultation platform (Communications NHS Forth Valley 2019).

\subsubsection{Field andCommunity Screening: Smartphone Glaucoma Screening in Kenya}

In 2013-2014, the London School of Hygiene and Tropical Medicine, in collaboration with the University of Strathclyde and NHS Forth Valley, performed a validation study to compare store- andforward teleophthalmology for the grading of optic nerve images captured with a handheld smartphone- 
based retinal imaging adaptor, with those of a reference fundus camera. In particular, more than 2000 images were captured over 100 population clusters in Kenya between 2013 and 2014 and transmitted to the Moorfield Eye Hospital Reading Centre, in the UK, to be independently graded (Bastawrous et al. 2016).

The examinations in Kenya were performed in field clinics, after pupil dilation, using the smartphone-based retinal imager and a reference benchtop fundus camera by two independent examiners when possible. Both experienced ophthalmologists and lay operators, with no prior experience of healthcare, were used for the imaging. The images were subsequently uploaded from Kenya to the Moorfields Reading Centre in London using a custom software platform. The study showed a comparable clinical performance between handheld retinal imaging and traditional fundus photography in the grading of the optic disk images for glaucoma.

Importantly, there was no observable difference in clinical readability between the images taken by experienced ophthalmologists and lay community operators. Indeed, the non-clinical photographers using the low-cost smartphone imaging adapter were able to acquire optic nerve images that enabled grading to the same clinical standard of the images acquired using the desktop retinal camera operated by an ophthalmic assistant. The principle of employing lay patientside operators to task-shift smartphone-based teleophthalmology from secondary care to the field appeared therefore well posed, at least for glaucoma screening, making it attractive for public health interventions.

\subsubsection{Optometric Decision Support: Australia and Scotland}

Using a combination of store-and-forward and realtime teleophthalmology, investigators in Australia (Bartnick et al. 2018) retrospectively audited the makeup of the referrals over a 12-month period, encompassing 683 remote consultations connecting optometry with hospital eye services in Western Australia. Equipment of referring optometric practices varied widely, from slit lamp alone, to more extensive setups with OCT, visual fields and wideangle retinal photography capacity.

The referrals represented a mix of scheduled consultations and emergency decision support. Over the year-long period of evaluation, the authors deemed that 287 patients were managed effectively via teleophthalmology, hence saving ten full-day outreach clinic days, expediting cataract surgery for those in rural settings via direct bookings from teleophthalmology consultations.

Within Central Scotland, extending the paradigm outlined in 4.1 to primary care optometric practices, the high-fidelity screen mirroring functionality of the NHS browser-based telemedicine platform (NHS Near Me 2019) has also been leveraged to cascade live digital data towards realtime decision support and enhanced triage. At the time of writing, four optometric practices within the NHS Forth Valley community, in a Scottish Government funded trial (presently unpublished), have collectively referred to secondary care, more than 70 consecutive patients over a 9-month period. Live video slit lamp imaging of anterior and posterior segment, as well as OCT volume scans and ultra-wide field images are mirrored directly from community practices to ophthalmology, complemented by webcams for face-to- face patient counselling. Although data collection is in progress, secondary care appointments to date have been judged by optometrist and ophthalmologist to be obviated between $40 \%$ and $50 \%$ of cases, with high indices of patient, optometrist and clinician satisfaction.

\subsubsection{Digital Wards: Virtual Clinics}

In teleophthalmology models of care, a prevalence of scheduled, namely, outpatient screening, general services and disease-specific consultations, as opposed to unscheduled (emergency and preoperatory) care is observed. These models are typically structured around a collaboration between local and remote care providers (ophthalmologists, optometrists, community care) (Caffery et al. 2019). Yet, scheduled care indeed proceeds along a paradigm whereby patients are seen in groups, in physician-led clinics. Unsurprisingly, as previously mentioned, existing large-scale telemedicine software infrastructure enables virtualisation of patient pathways modelled along in-person clinics, with virtual waiting areas, virtual queues, etc. (Attend Anywhere 2019; NHS Near Me 2019). Indeed, as for 2014, $42 \%$ of US hospitals offered 
some form of outpatient virtual clinics (AdlerMilstein et al. 2014).

In the specific case of teleophthalmology, evidence base is accumulating on the fact that, at least from the point of view of immediate clinical outcomes, this practice is essentially safe, with remote visualisation-based clinics demonstrated for diabetic retinopathy, glaucoma, anterior segment diseases, non-diabetic retinopathies and more (Caffery et al. 2019).

Yet, telemedicine suffers from potential unintended consequences related to the change in relations, both interpersonal and interprofessional, between all stakeholders involved. Patients, physicians, nurses and allied health professionals are effectively asked to establish a professional and interpersonal trust, rapport and organisational hierarchy with counterparts who they have potentially never met in person, impacting on cultural, organisational and socioeconomic correlates of healthcare provision (Harrison et al. 2007), with poorly understood, yet potentially farreaching and disruptive impacts (Kahn 2015).

\section{$9.5 \quad$ Outlook}

The ability of computerised systems to carry out tasks that we normally associate with intelligence (Artificial Intelligence, AI) or, more specifically, machine learning, especially in the embodiments that rely on massive datasets of template examples used to optimise the performance of computer algorithms, broadly described as 'deep learning', are opening new pathways to medicine. In particular, they are enabling automated systems to extract accurate diagnostic interpretations from patient data, most often image-based. Further medical AI applications touch upon careflow optimisation and consumer-end processing of healthcare data for healthcare promotion such as, for example, in mainstream consumer fitness monitors, or more recently, in full regulatory- compliant diagnostic tools based on consumer devices (Topol 2019).

The most robust medical AI implementations revolve around imaging-intensive medical specialities, in primis radiology, pathology and dermatology. Teleophthalmology relies critically on realtime or deferred remote visualisation of images or videos collected at the patient site. For this reason, teleophthalmology appears as ideal candidate for AI. Indeed, in digital ophthalmology, effective algorithms have been demonstrated for the detection of disease from retinal images (diabetic retinopathy, glaucoma, age-related macular degeneration, retinopathy of prematurity, refractive error, cardiovascular risk factors) and OCT scans (several macular conditions, diabetic macular oedema, early stages of AMD, choroidal neovascular membrane) (Ting et al. 2019).

Models of care for AI-enhanced telemedicine envisage the AI engine to be deployed either as a cloud-based platform or on one of the imaging end points, arguably reducing the need for highresolution image transfer and for the related highdata-rate digital infrastructure, hence better suited in low-resource settings. Indeed, low-cost low-power AI-dedicated processing platforms are now available from mainstream vendors (Intel Movidius 2019; Coral 2019; nVidia Corp 2019) enabling imagingbased inference algorithms to be implemented on handheld devices at a price point compatible with mass distribution.

Yet, this all is not without risk. Indeed, AI algorithms are 'black boxes' delivering an apparently consistent output from raw data input, on the basis of a large set of template examples. The closer the machine behaviour is to a 'black box' mimicking human intelligence, with no clear sight of the internal workings, the higher the risk of physician deskilling, loss of context in favour of data, loss of perception of the intrinsic uncertainty of medical findings (Cabitza et al. 2017), bias, privacy and security breaches, lack of transparency (Topol 2019), susceptibility to attack and manipulation and lack of regulatory accountability (Kelly et al. 2019), just to mention a few of the risk factors emerging from recent literature. It is the belief of the authors of this chapter that remote visualisation-based teleophthalmology will affect clinical practice, whether with or without AI support, and the potential for AI-enhanced clinical value improvement must be balanced against the risk of negative outcomes on a pragmatic basis.

Innovative approaches are emerging also on the development of teleophthalmology-dedicated hardware. In particular, teleophthalmology platforms have been demonstrated whereby remote slit lamp visualisation is complemented by remote actuation of the slit lamp position, resulting in an 
effective robotic platform, entirely teleoperated by the ophthalmologist (Chatziangelidis 2014). More recently, a robotised slit lamp platform has been integrated with full stereoscopic visualisation, and tested over a long-distance satellite data link (Nankvil et al. 2018).

Indeed, teleophthalmology is entering a new, exciting development stage, as ideal platform where medicine, system and telecommunications engineering, computer science and robotic technologies converge for remote visualisation, AI and remote actuation to express their full integration potential.

\section{References}

Adler-Milstein J, Kvedar J, Bates DW (2014) Telehealth among US hospitals: several factors, including state reimbursement and licensure policies. Influence Adop Health Aff 33:207-215. https://doi.org/10.1377/ hlthaff.2013.1054

Ahmed SM, Adams AM, Chowdhury M et al (2000) Gender, socioeconomic development and healthseeking behaviour in Bangladesh. Social Sci Med 51:361-371

AMD Global Telemedicine (2019) Telemedicine technologies: real-time versus store and forward. Available at https://www.amdtelemedicine.com/blog/article/ telemedicine-technologies-real-time-versus-store- andforward. Accessed 31 Oct 2019

Attend Anywhere, Australia. https://attendanywhere.com. Accessed 13 Dec 2019

Bartnik SE, Copeland SP, Aicken AJ, Turner AW (2018) Optometry-facilitated teleophthalmology: an audit of the first year in Western Australia. Clin Exp Optom 101:700703. https://doi.org/10.1111/cxo.12658

Bass SJ (2009) Examination of the posterior segment of the eye. In: Rosenfield M, Logan N (eds) Optometry, 2nd edn. Butterworth, pp 277-298

Bastawrous A (2014) Get your next eye exam on a smartphone. TED. Available at https://www.ted.com/talks/ andrew_bastawrous_get_your_next_eye_exam_ on_a_smartphone. Accessed 13 Dec 2019

Bastawrous A, Hennig BD (2012) The global inverse care law: a distorted map of blindness. Br J Ophthalmol 96:13571358. https://doi.org/10.1136/ bjophthalmol-2012-302088

Bastawrous A, Giardini ME, Bolster NM et al (2016) Clinical validation of a smartphone-based adapter for optic disc imaging in Kenya. JAMA Ophthalmol 134:151-158. https://doi.org/10.1001/ jamaophthalmol.2015.4625

Blencowe H, Lawn JE, Vazquez T et al (2013) Pretermassociated visual impairment and estimates of retinopathy of prematurity at regional and global levels for 2010 . Pediatr Res 74:35-49. https://doi. org/10.1038/pr.2013.205

Bolster NM, Giardini ME, Livingstone IAT, Bastawrous A (2014) How the smartphone is driving the eye-health imaging revolution. Exp Rev Ophthalmol 9:475-485. https://doi.org/10.1586/17469899.2014.981532

Cabitza F, Rasoini R, Gensini GF (2017) Unintended consequences of machine learning in medicine. JAMA 318:517-518. https://doi.org/10.1001/ jama.2017.7797

Caffery LJ, Taylor M, Gole G, Smith AC (2019) Models of care in tele-ophthalmology: a scoping review. J Telemed Telecare 25(2):106-122. https://doi.org/10.1 177/1357633X17742182

Carl Zeiss Meditec AG (n.d.) Eye Examination with the Slit Lamp. Publication no. 000000-1152-355

Chatizangelidis I (2014) Construction of low cost remote controlled slit lamp (R/C/S/L) in order to provide teleophthalmology services to rural areas and islands. Adv Ophthalmol Vis Syst 1:00025. https://doi. org/10.15406/aovs.2014.01.00025

Clewley D, Rhon D, Flynn T et al (2018) Health seeking behaviour as a predictor of healthcare utilization in a population of patients with spinal pain. PLoS One 13: 0201348 . pone. 0201348

Communications NHS Forth Valley (2019) World's first 5G tele-examination of an eye. Available from: https:// nhsforthvalley.com/worlds-first-5g-tele-examination- ofan-eye/. Accessed 10 Dec 2019

Coral. https://coral.ai. Accessed 13 Dec 2019

D-Eye, Italy. D-Eye retina. https://www.d-eyecare.com/. Accessed 1 Nov 2019

Ebeigbe JA (2018) Factors influencing eye-care seeking behaviour of parents for their children in Nigeria. Clin Exp Optom 101:560-564. https://doi.org/10.1111/ cxo. 12506

Eidolon Optical, USA: Photo Bluminator 2. https://www. slitlamp.com/photo-bluminator-ii. Accessed 1 Nov 2019

Fallatah MO (2018) Knowledge, awareness, and eye careseeking behavior in diabetic retinopathy: a crosssectional study in Jeddah, Kingdom of Saudi Arabia. Ophhthalm Ther 7:377-385. https://doi.org/10.1007/ s40123-0180147-5

Fleming AD, Philip S, Goatman KA et al (2011) The evidence for automated grading in diabetic retinopathy screening. Curr Diab Rev 7:246-252. https://doi. org/10.2174/157339911796397802

Freitas AM, Morschbacher R, Thorell MR, Rhoden EL (2018) Incidence and risk factors for retinopathy of prematurity: a retrospective cohort study. Int J Retin Vitr 4:20. https://doi.org/10.1186/s40942-018-0125-z

Giardini ME (2015) The portable eye examination kit: mobile phones cand screen for eye disease in low-resource settings. IEEE Pulse 2015 Nov/Dec. Available at https://pulse.embs.org/november-2015/ the-portable-eyeexamination-kit. Accessed 13 Dec 2019

Giardini ME, Livingstone IAT, Jordan S et al (2014) A smartphone based ophthalmoscope. Conf Proc IEEE Eng Med Biol Soc 2014:2177-2180. https://doi. org/10.1109/EMBC.2014.6944049

GrisoliaABD,Abalem F, LuY et al (2017)Teleophthalmology: where are we now? Arq Bras Oftalmol 80:401-405. https://doi.org/10.5935/0004-2749.20170099

GSM Association (2019) The mobile economy 2019. Available at https://www.gsma.com/r/mobileecon- omy/. Accessed 13 Dec 2019

Harrison MI, Kppel R, Bar-Lev S (2007) Unintended consequences of information technologies in health care - an 
interactive sociotechnical analysis. J Am Med Inform Assoc 14:542-549. https://doi.org/10.1197/ jamia.M2384

Hellstrom A, Smith LEH, Dammann O (2013) Retinopathy of prematurity. $\quad$ Lancet 382:1445-1457. https://doi.org/10.1016/S0140-6736(13)60178-6

Holmstrom H, Tornqvist K, Al-Hawasi A et al (2018) Increased frequency of retinopathy of prematurity over the last decade and significant regional differences. Acta Ophthalmol 96:142-148. https://doi. org/10.1111/aos.13549

Intel Movidius. https://www.movidius.com/. Accessed 13 Dec 2019

Jonuscheit S (2019) General ophthalmic services in Scotland: value for (public) money? Ophth Phys Opt 39:225-231. https://doi.org/10.1111/opo.12632

Kahn JM (2015) Virtual visits - confronting the challenges of telemedicine. N Engl J Med 372:1684-1685

Keeler CR (2003) A brief history of the ophthalmoscope. Optometry Prac 4:137-145

Kelly SP, Wallwork I, Haider D, Qureshi K (2011) Teleophthalmology with optical coherence tomography imaging in community optometry. Evaluation of a quality improvement for macular patients. Clin

Ophthalmol 5:1673-1678. https://doi.org/10.2147/ OPTH.S26753

Kelly CJ, Karthikesalingam A, Suleyman M et al (2019) Key challenges for delivering clinical impact with artificial intelligence. BMC Med 17:195. https://doi. org/10.1186/s12916-019-1426-2

Lapere S (2018) Tele-ophthalmology for the monitoring of choroidal and iris nevi: a pilot study. Can J Ophthalmol 53:471-473. https://doi.org/10.1016/j. jcjo.2017.11.021

Lee JX, Manjunath V, Talks SJ (2018) Expanding the role of medical retina virtual clinics using multimodal ultrawidefield and optical coherence tomography imaging. Clin Ophthalmol 12:2337-2345. https://doi. org/10.2147/OPTH.S181108

Loane MA, Bloomer SE, Corbett R et al (2008) A comparison of real-time and store-and-forward teledermatology: a cost-benefit study. BJD 143:1241-1247. https://doi.org/10.1046/j.1365-2133.2000.03895.x

Loomba A, Vempati S, Davara ND et al (2019) Use of a tablet attachment in teleophthalmology for realtime video transmission from rural vision centers in a three-tier eye care network in India: eyeSmart cyclops. Int J Telemed Appl 5683085. https://doi. org/10.1155/2019/5683085

Mackian S, Bedri N, Lovel H (2004) Up the garden path and over the edge: where might health-seeking behaviour take us? Health Policy Plan 19:137-146. https:// doi.org/10.1093/heapol/czh017

Nankvil D, Gonzalez A, Rowaan C et al (2018) Robotic controlled stereo slit lamp. TVST 7:1-13. https://doi. org/10.1167/tvst.7.4.1

National Center for Connected Health Policy (2019a) Storeand-forward (asynchronous). https://www. cchpca.org/about/about-telehealth/store-and-forwardasynchronous. Accessed 31 Oct 2019

National Center for Connected Health Policy (2019b) Current state laws \& reimbursement policies. https:// www.cchpca.org/telehealth-policy/current-state-lawsand-reimbursement-policies. Accessed 31 Oct 2019

NHS Near Me, UK. https://nhsh.scot/nhsnearme. Accessed 13
Dec 2019

nVidia Corp (2019) Jetson Nano. https://wwwnvidiacom/ engb/autonomous-machines/embedded-systems/jet- sonnano/. Accessed 13 Dec 2019

oDocs Eye Care, New Zealand (2019a) visoScope. https:// www.odocs-tech.com/visoscope/\#. Last visited 1 November 2019

oDocs Eye Care, New Zealand (2019b) Fundus. http:// www.odocs-tech.com/fundus/\#. Last visited 1 November 2019

Owens B (2018) Telemedicine on the rise but lagging in Canada. CMAJ 190:E1149-E1150. https://doi. org/10.1503/cmaj.109-5634

Peek Vision Ltd., UK. Peek Retina. https://www. peekvision.org/en_GB/peek-solutions/peek-retina/. Accessed 13 Dec 2019

Poyser O, Livingstone I, Ferguson A et al (2019) Realtime Tele-ophthalmology in the emergency department. IOVS 60:6124

Rani PK, Raman R, Manikandan M et al (2006) Patient satisfaction with tele-ophthalmology versus ophthalmologist-based screening in diabetic retinopathy. J Telemed Telecare 12:159-160

Resnikoff S, Felch W, Gauthier T-M et al (2012) The number of ophthalmologists in practice and training worldwide: a growing gap despite more than 200,000 practitioners. Br J Ophthalmol 96:783-787. https:// doi.org/10.1136/bjophthalmol-2011-301378

Rho MJ, Choi IY, Lee J (2014) Predictive factors of telemedicine service acceptance and behavioural intention of physicians. Int J Med Inform 83:559-571. https:// doi.org/10.1016/j.ijmedinf.2014.05.005

Ribeiro AG, Rodrigues RAM, Guerreiro AM, Regatieri CVS (2014) A teleophthalmology system for the diagnosis of ocular urgency in remote areas of Brazil. Arq Bras Oftalmol 77:214-218. https://doi. org/10.5935/00042749.20140055

Shahik BT, Hatcher J (2004) Health seeking behaviour and health service utilization in Pakistan: challenging the policy makers. J Public Health 27:49-54. https:// doi.org/10.1093/pubmed/fdh207

Sim DA, Mitry D, Alexander P et al (2016) The evolution of teleophthalmology programs in the United Kingdom: beyond diabetic retinopathy screening. J Diabetes Sci Technol 10:308-317. https://doi. org/10.1177/1932296816629983

Sreelatha OK, Ramesh SVS (2016) Teleophthalmology: improving patient outcomes? Clin Ophthalmol 10:285295. https://doi.org/10.2147/OPTH.S80487

Surendran TS, Raman R (2014) Teleophthalmology in diabetic retinopathy. J Diab Sci Technol 8:262-266. https://doi.org/10.1177/1932296814522806

The College of Optometrists (2014) A strategy to improve ophthalmic public health 2014. Available at https:// www.college-optometrists.org/resourceLibrary/astrategy-to-improve-ophthalmic-public-health-2014. html. Accessed 31 Oct 2019

Timoney PJ, Breathnach CS (2013) Alvar Gullstrand and the slit lamp 1911. Ir J Med Sci 182:301-305. https:// doi.org/10.1007/s11845-012-0873-y

Ting DSW, Peng L, Varadarajan AV et al (2019) Deep learning in ophthalmology: the technical and clinical 
considerations. Prog Retin Eye Res 72:100759. https://doi.org/10.1016/j.preteyeres.2019.04.003

Topol EJ (2019) High-performance medicine: the convergence of human and artificial intelligence. Nat Med 25:44-56. https://doi.org/10.1038/s41591-018-0300-7

Tuteja SY. The Arclight: A 'pocket' ophthalmoscope to revitalise undergraduate teaching? Eye News, 1 Dec 2016. Available from: https://www.eyenews.uk.com/ education/trainees/post/the-arclight-a-pocket-ophthalmoscope-to-revitalise-undergraduate-teaching. Accessed 13 Dec 2019

UBORA: Euro-African open biomedical engineering eplatform for innovation through education. http:// uborabiomedical.org/. Accessed 13 Dec 2019

United Nations (2019) World population prospects 2019. https://population.un.org/wpp/Publications/. Accessed 13 Dec 2019

Volk. iNview. https://volk.com/index.php/volk-products/ ophthalmic-cameras/volk-inview.html. Accessed 13 Dec 2019

Welch-Allyn, USA (2019a) PanOptic Ophthalmoscope. https://www.welchallyn.co.uk/content/welchallyn/ emeai/uk/products/categories/physical-exam/eyeexam/ophthalmoscopes\%2D\%2Dwide-view-direct/ panoptic_ophthalmoscope.html. Accessed 1 Nov 2019

Welch-Allyn, USA (2019b) iEXAMINER. https:// www.welchallyn.com/en/microsites/iexaminer.html. Accessed 1 Nov 2019

World Health Organization (2018) Ageing and health. Available at https://www.who.int/news-room/factsheets/detail/ageing-and-health. Accessed 13 Dec 2019

World Health Organization (2019a) Priority eye diseases. Available at https://www.who.int/blindness/causes/ priority/en/. Accessed 13 Dec 2019

World Health Organization (2019b) Guideline "Recommendations on digital interventions for health system strengthening". Available at https://www.who. int/reproductivehealth/publications/digital-interven- tionshealth-system-strengthening/en/. Accessed 12 Dec 2019

Yu P, Li H, Gagnon MP (2009) Health IT acceptance factors in long-term care facilities: a cross-sectional survey. Int J Med Inform 78:219-229. https://doi. org/10.1016/j.ijmedinf.2008.07.006 